\title{
A prática colaborativa na formação em saúde: Relato de experiência do PET saúde
}

\section{Interprofissionalidade}

\author{
Collaborative practice in health education: Experience report of PET health Interprofessionality \\ Práctica colaborativa en educación para la salud: Informe de experiencia del PET salud \\ interprofesionalidad
}

Recebido: 01/04/2021 | Revisado: 08/04/2021 | Aceito: 14/04/2021 | Publicado: 29/04/2021

Suzana de Oliveira Mangueira

ORCID: https://orcid.org/0000-0003-0931-8675

Universidade Federal de Pernambuco, Brasil

E-mail: suzana.mangueira@ufpe.br

Érika Michelle Correia de Macedo

ORCID: https://orcid.org/0000-0002-9017-5191

Universidade Federal de Pernambuco, Brasil E-mail: erika.macedo@ufpe.br

Vera Lúcia Rodrigues de Albuquerque ORCID: https://orcid.org/0000-0002-1531-1819 Secretaria Municipal de Saúde de Vitória de Santo Antão, Brasil

E-mail: albuquerque.vera37@gmail.com Mônica Cabral Pereira

ORCID: https://orcid.org/0000-0002-3240-5032 Secretaria Municipal de Saúde de Vitória de Santo Antão, Brasil E-mail: edufisica68@gmail.com

Emanuelly Carollyne Lopes Nascimento ORCID: https://orcid.org/0000-0002-5903-5561 Secretaria Municipal de Saúde de Vitória de Santo Antão, Brasil

E-mail: lopes.emanuelly94@gmail.com

Maria Angélica Álvares de Freitas ORCID: https://orcid.org/0000-0002-2680-909X Secretaria Municipal de Saúde de Vitória de Santo Antão, Brasil

E-mail: angelica_alvares_2@hotmail.com

Gledson Wilber de Souza

ORCID: https://orcid.org/0000-0003-3258-3143

Universidade Federal de Pernambuco, Brasil

E-mail: gledson_wilber@hotmail.com

Ana Luiza Lacerda de Souza Pinheiro

ORCID: https://orcid.org/0000-0001-9071-2930

Universidade Federal de Pernambuco, Brasil

E-mail: nutrianaluizalacerda@gmail.com

Felipe Maia Tardieux

ORCID: https://orcid.org/0000-0001-8275-1243

Universidade Federal de Pernambuco, Brasil

E-mail: felipemaiatardieux@gmail.com

Joanna Beatriz de Oliveira Silva

ORCID: https://orcid.org/0000-0002-9852-6558

Universidade Federal de Pernambuco, Brasil E-mail: beatrizjoanna4@gmail.com

Luciano da Silva Medeiros Júnior

ORCID: https://orcid.org/0000-0002-2109-1410

Universidade Federal de Pernambuco, Brasil E-mail: lucianojr.ldsmj@gmail.com

Danielly Alves Mendes Barbosa

ORCID: https://orcid.org/0000-0003-4540-0334

Universidade Federal de Pernambuco, Brasil E-mail: danielly.alvesmb@gmail.com

Caroline Silva dos Santos

ORCID: https://orcid.org/0000-0001-7746-4733

Universidade Federal de Pernambuco, Brasil

E-mail: carolinesnutricionista@gmail.com 


\begin{abstract}
Resumo
Objetivo: Relatar a experiência de um grupo de aprendizagem tutorial do PET Saúde Interprofissionalidade, com vistas à promoção da prática colaborativa na formação em saúde. Método: Trata-se de um relato de experiência de um grupo de aprendizagem tutorial do projeto PET Saúde Interprofissionalidade em um município do estado de Pernambuco. A equipe conta com tutores docentes, preceptores profissionais do serviço e estudantes de graduação e residência, contemplando os seguintes cursos: educação física, enfermagem, nutrição, psicologia, saúde coletiva e serviço social. São realizadas reuniões semanais para aprofundamento teórico, elaboração de material educativo e discussão de casos complexos, bem como visitas domiciliares às famílias em acompanhamento. Resultados: No ano de 2020, realizou-se o acompanhamento sistemático de famílias com problemas principais de obesidade e sofrimento psíquico. A cada semana, as visitas domiciliares eram realizadas por um subgrupo de preceptores e discentes, para avaliar e intervir junto às famílias. Os casos eram discutidos nas reuniões, nas quais eram elaboradas as propostas de intervenção em conjunto, por meio da prática colaborativa. Conclusão: Observou-se que o projeto promoveu a integração do ensino-serviço-comunidade, proporcionou aos estudantes a experiência da prática colaborativa interprofissional na sua formação, bem como um atendimento individualizado e de qualidade às famílias assistidas.

Palavras-chave: Educação interprofissional; Capacitação de recursos humanos em saúde; Práticas interdisciplinares; Saúde pública.
\end{abstract}

\begin{abstract}
Objective: To report the experience of a tutorial learning group from PET Health Interprofessionality, with a view to promoting collaborative practice in health education. Method: This is an experience report of a tutorial learning group from the PET Health Interprofessionality project in a municipality in the state of Pernambuco. The team includes teaching tutors, professional from the service preceptor and undergraduate and residency students, covering the following courses: physical education, nursing, nutrition, psychology, public health and social work. Weekly meetings are held for theoretical study, preparation of educational material and discussion of complex cases, as well as home visits to families being monitored. Results: In 2020, families with major problems of obesity and psychological distress were systematically monitored. Each week, home visits were carried out by a subgroup of tutors and students, to assess and intervene with families. The cases were discussed at the meetings, in which joint intervention proposals were prepared, through collaborative practice. Conclusion: It was observed that the project promoted the integration of teaching-service-community, provided students with the experience of collaborative interprofessional practice in their training, as well as individualized and quality care to assisted families.
\end{abstract}

Keywords: Interprofessional education; Health human resource training; Interdisciplinary placement; Public health.

\title{
Resumen
}

Objetivo: Informar la experiencia de un grupo de aprendizaje tutorial de PET Saúde Interprofesionalidad, con miras a promover la práctica colaborativa en educación para la salud. Método: Este es un relato de experiencia de un grupo de aprendizaje tutorial del proyecto PET Saúde Interprofesionalidad en un municipio del estado de Pernambuco. El equipo está integrado por tutores docentes, tutores profesionales del servicio y estudiantes de pregrado y residencia, cubriendo los siguientes cursos: educación física, enfermería, nutrición, psicología, salud pública y trabajo social. Se realizan reuniones semanales de estudio teórico, elaboración de material educativo y discusión de casos complejos, así como visitas domiciliarias a las familias en seguimiento. Resultados: En 2020 se monitoreó sistemáticamente familias con grandes problemas de obesidad y angustia psicológica. Cada semana, un subgrupo de tutores y estudiantes realizaba visitas domiciliarias, para evaluar e intervenir con las familias. Los casos fueron discutidos en las reuniones, en las que se elaboraron propuestas de intervención conjunta, a través de la práctica colaborativa. Conclusión: Se observó que el proyecto promovió la integración enseñanza-servicio-comunidad, brindó a los estudiantes la experiencia de la práctica interprofesional colaborativa en su formación, así como una atención individualizada y de calidad a las familias asistidas.

Palabras clave: Educación interprofesional; Capacitación de recursos humanos en salud; Prácticas interdisciplinarias; Salud pública.

\section{Introdução}

O tema interprofissionalidade tem mobilizado, atualmente, educadores, pesquisadores, órgãos e instituições de saúde e educação, e vem ganhando contornos de um movimento com a realização de projetos e eventos, e na criação de instituições, como a Rede Regional de Educação Interprofissional das Américas (REIP). Por outro lado, existem ainda muitos limites e resistências teórico-práticas presentes na educação interprofissional em saúde, devido a fortes domínios profissionais e curriculares inundados pelo individualismo disciplinar especializado (Lima et al, 2020).

No Brasil, o modelo de formação dos profissionais de saúde ainda acontece de forma uniprofissional e disciplinar. Um modelo no qual as práticas isoladas não favorecem o trabalho em equipe subjetiva o usuário, categoriza o cuidado e atenua 
ainda mais o atraso com as difíceis necessidades do mundo atualmente, que se torna cada vez mais afastada de realidades e contextos da prática (Costa et al, 2018; Costa, 2016). Por isso, é necessário o levantamento de respostas para questões, por exemplo, sobre como é possível avançar na educação interprofissional em saúde, caracterizada por uma estrutura formativa centrada na doença e nas técnicas, e não no usuário, com o intuito de auxiliar no desenvolvimento das políticas de Educação e Saúde.

Dá-se por Educação Interprofissional (EIP) aquela em que estudantes de duas ou mais profissões aprendem sobre os outros, com os outros e entre si para possibilitar a efetiva colaboração e melhorar os resultados na saúde (OMS, 2010). Tal estratégia tem como objetivo oferecer oportunidades de formação conjunta com o intuito de desenvolver atributos e competências para a prática colaborativa, repercutir em uma atenção às necessidades de saúde eficaz, integral e universal. Acredita-se que a experimentação e o conviver interativo são essenciais para o desenvolvimento de competências colaborativas (Riskiyana et. al, 2018).

Ao longo dos anos, estudos destacam o desenvolvimento de competências colaborativas para o trabalho em equipe como: identificação e reconhecimento dos papeis profissionais com mais respeito entre tais categorias, melhor comunicação entre os membros da equipe e compreensão da interdependência entre todos os membros da equipe, com vistas a favorecer a complementaridade, a qualidade e a segurança do cuidado (Guraya \& Barr, 2018). O Sistema Único de Saúde (SUS) tem sido referência para as mudanças do trabalho e da formação em saúde e para o desenvolvimento de tais competências através da prática contínua e da colaboração interprofissional presente em ações estratégicas como o trabalho em Equipes de Saúde da Família (ESF), de Agentes Comunitários de Saúde (ACS) e como a formação em Programas de Educação pelo Trabalho na saúde (PET-Saúde).

Nesta vertente, em 2018, o Ministério da Saúde publicou o edital de seleção para o Programa de Educação pelo Trabalho para a saúde PET Saúde/Interprofissionalidade, voltado a integração ensino-serviço-comunidade, de forma articulada entre o SUS e as instituições de ensino, de modo a promover a EIP e as Práticas Colaborativas em Saúde. Os Grupos de Aprendizagem Tutorial (GAT) do projeto devem estar direcionados a estimular o desenvolvimento de competências colaborativas, devendo também demonstrar capacidade de estimular mudança curricular em cada curso envolvido, sem dispensar a discussão e desenvolvimento de competências específicas (complementares) e comuns nos processos de mudança dos cursos entre si.

A Universidade Federal de Pernambuco / Centro Acadêmico de Vitória, em parceria com a secretaria de saúde do município, foi contemplado com a aprovação de um projeto no referido edital e desenvolve as atividades desde 2019. A realização de capacidades colaborativas necessita de prática contínua e de colaboração interprofissional. Considerando esse contexto, este estudo teve como objetivo relatar a experiência de um grupo de aprendizagem tutorial do PET Saúde Interprofissionalidade, com vistas à promoção da prática colaborativa na formação em saúde.

\section{Metodologia}

Trata-se de um estudo descritivo, tipo relato de experiência, elaborado no contexto do Programa de Educação pelo Trabalho para a Saúde (PET Saúde) Interprofissional, atuante no município Vitória de Santo Antão, Pernambuco. Os participantes do projeto incluem dois tutores, professores da UFPE, com formação nas áreas de enfermagem e nutrição; quatro preceptores profissionais do serviço, sendo uma enfermeira, uma nutricionista, uma assistente social e uma profissional de educação física; cinco estudantes de graduação dos cursos de enfermagem, nutrição, educação física e saúde coletiva e dois estudantes de residência multiprofissional, sendo um psicólogo e uma nutricionista. O projeto foi desenvolvido no período de 2019 a 2021 e foi selecionado o ano de 2020 para a elaboração deste relato de experiência.

Inicialmente, a equipe foi treinada, para alinhamento teórico e conceitual sobre a Educação Interprofissional, por meio 
da discussão de textos e realização de cursos. Na sequência, os preceptores, profissionais do serviço, apresentaram o território de atuação, que abrangeu duas equipes de saúde da família apoiadas por uma mesma equipe de Núcleo de Apoio à Saúde da Família (NASF), nas comunidades de Redenção e Lídia Queiroz, bairros do município de Vitória de Santo Antão - PE. Os preceptores eram responsáveis ainda por selecionar as famílias a serem acompanhadas e orientar as atividades presenciais dos alunos no serviço.

No ano de 2020 foi realizado o acompanhamento de famílias. Entre os problemas enfrentados, que foram selecionados como prioridade pelo projeto para a realização de intervenções, destacam-se a obesidade e sofrimento psíquico, com ênfase na depressão e abuso de substâncias. Desta forma, a equipe de preceptores e estudantes se dividia em subgrupos e realizavam o acompanhamento semanal ou quinzenal das famílias por meio de visitas domiciliares. Nestas visitas, era feita a escuta da família, orientações e encaminhamentos.

Eram realizadas reuniões semanais com toda a equipe, mediada pelos tutores, para discussão dos casos e propostas de intervenções, que incluíram avaliação e orientações nutricionais, avaliação física e plano de atividade física dentro dos limites e possibilidades das famílias, auriculoterapia, orientações sobre as medicações utilizadas e encaminhamentos para serviços de saúde (médico, psicólogo, dentista). Todas as atividades eram registradas no relatório de cada família, com avaliação da classificação de risco (Coelho \& Savassi, 2012), elaboração de genograma e ecomapa, além das intervenções realizadas e a avaliação. O material oriundo destes relatos subsidiou a elaboração dos relatórios do projeto e trabalhos/artigo científicos.

Quanto aos aspectos éticos, destaca-se que este estudo trata-se de um relato de experiência de atividade de caráter extensionista e não de atividade de pesquisa. Dessa forma, não houve submissão ao Comitê de Ética em Pesquisa.

\section{Resultados e Discussão}

A partir das visitas e acompanhamentos realizados pela equipe interprofissional nas comunidades de Redenção e Lídia Queiroz, bairros do município de Vitória de Santo Antão - PE foi possível identificar algumas demandas, para as quais foram aplicadas intervenções pelo grupo e sendo possível obter alguns resultados positivos.

Inicialmente, todo o trabalho e intervenções se deram a partir de reuniões da equipe PET e Estratégia de Saúde família (ESF), em que foi possível mapear famílias que apresentavam maior vulnerabilidade social, de modo a identificar suas necessidades de saúde. Trabalho semelhante foi realizado por um PET, na atenção primária em um Centro de Saúde da Família no município de Chapecó-SC, onde há uma grande população de baixa renda em um território industrial e foi apresentado pelos Agentes Comunitários de Saúde (ACS) como particularidades da área o alto índice de gravidez na adolescência e drogadição, sendo possível intervir em equipe nessas situações (Machado et al, 2019).

Diferente das intervenções realizadas por esse grupo de Chapecó-SC, que faz suas intervenções em uma escola, local onde está o maior público de adolescente, as intervenções do projeto foram feitas na casa dos comunitários, no entanto, apresentam como objetivo final em comum o melhor acolhimento para o usuário e a resolução de seus problemas de modo integral. Este tipo de atividade busca a valorização de todos profissionais da equipe, compartilhando conhecimento e estreitando laços, além disso, proporciona que o acadêmico assuma um papel de sujeito transformador na construção de uma visão de saúde ampliada, com fortalecimento da atuação baseada na humanização e pautada na constante reflexão acerca da interdisciplinaridade (Montenegro, 2013).

Consideravelmente, foi possível detectar ainda uma aproximação dos comunitários com as redes de atenção disponíveis no município, como o Centro de Apoio Psicossocial (CAPS), Centro de Referência de Assistência Social (CRAS) e com a Unidade de Saúde. Especificamente, na Unidade de Saúde, foi possível proporcionar momentos de troca de ideias e experiências, para que a população pudesse junto com a equipe esclarecer suas dúvidas e partilhar conhecimento. Essa articulação proporcionou a construção de estratégias de cuidado compartilhado, auxiliando na resolubilidade da atenção e 
criou-se uma co-responsabilização pelos casos, por meio de discussões e intervenções conjuntas, junto às famílias. Isto acarreta a construção de sujeitos cada vez mais autônomos, por ajudar na compreensão do indivíduo sobre o seu processo saúdedoença, as relações sociais que influenciam tal processo e os recursos potenciais para melhoria de suas condições de vida, que, segundo Gazignato e Silva (2014), este deve ser o foco das equipes de saúde da família.

Encontrou-se, durante as visitas aos comunitários, alguns que apresentavam maior vulnerabilidade de entendimento e uso incorreto de seus fármacos, e foi instruído o uso correto por meio de tabelas com o nome de cada medicação e horário, deixando expostos em locais de fácil acesso dentro de suas residências, com vistas a um melhor resultado, de modo a evitar interações medicamentosas e os riscos da automedicação inadequada. Estas ações de educação em saúde corroboram com os achados de Wiese et al (2020), cujo trabalho relata as atividades de um projeto de extensão que tinha como um dos objetivos promover a educação em saúde como maior instrumento junto à população de Joinville-SC, contribuindo para uma prática de autocuidado e uso racional de medicação, sendo possível identificar efeitos positivos quando o usuário entende seu processo de cuidado no contexto saúde-doença e torna-se sujeito do mesmo.

Igualmente, estudo de Soares et al (2009), mostrou que, para além do simples repasse de informações do profissional de saúde, as metodologias dinâmicas e participativas contribuem para estimulação do indivíduo e o tornam mais comprometido com seu processo de promoção da saúde, enfretamento e controle das doenças e seus agravos.

Outra atividade interventiva do projeto foi a auriculoterapia, implantada como prática integrativa na rotina de cuidado dos comunitários, para a qual observou-se uma boa aceitação e procura. A atividade referida torna-se complementar e efetiva no cuidado em saúde e está inserida na Política de Práticas Integrativas e Complementares do Sistema Único de Saúde (SUS). Além de ser uma técnica de baixo custo, a auriculoterapia propõe resultados imediatos e benefícios em diversos âmbitos como na redução de peso, sintomas de ansiedade, alteração de sono e melhora da qualidade de vida (Vilaça \& Coutinho, 2019; Novack et al, 2020). As práticas complementares em geral contribuem para a efetivação do cuidado integral e humanizado, devendo ser incentivada e potencializada no cotidiano do serviço no âmbito da atenção básica, com vistas à promoção de saúde e qualidade de vida da população (Ferreira, 2019).

Como intervenções junto às famílias, também foram realizadas avaliação e orientações nutricionais. Com base em Cervato-Mancuso et al (2016), as intervenções educativas nutricionais devem ultrapassar as raízes biomédicas e ter como base o indivíduo para o coletivo, e de um conhecimento científico para um novo conhecimento construído pela participação de todos. Diante disso, e entendendo a importância e eficácia das intervenções educativas, foram realizadas orientações para esclarecimento acerca de assuntos relacionados à saúde nutricional com os conceitos de sobrepeso e baixo peso por meio dos usuários. A equipe de nutrição, por meio de receitas fáceis e saudáveis, incentivou os comunitários a terem uma refeição que suprisse melhor suas necessidades básicas.

A equipe, durante as intervenções, também relatou sobre os benefícios dos exercícios físicos para prevenção de doenças e agravos à saúde, estimulou o estilo de vida ativo e o combate ao sedentarismo. As orientações consideraram as comorbidades, desempenho funcional, necessidade e limitações de cada usuário, com as devidas adequações. De modo similar, a equipe PET de Cuiabá-MT também investiu nas ações referentes a educação física, de igual forma observou que essas intervenções são eficazes para promover saúde, ofertar informações e estratégias para práticas corporais, intervindo positivamente sobre a qualidade de vida (Silva et al, 2012).

\section{Considerações Finais}

A relevância do projeto PET Saúde Interprofissionalidade é percebida a partir das experiências vivenciadas pelos diferentes atores envolvidos nesse processo de fazer saúde (alunos, professores, profissionais de saúde e comunitários). Diferentemente das intervenções pensadas apenas pela academia, ou apenas pelos profissionais de saúde, as ações do PET se 
propuseram a unir todas essas linhas de pensamento e intervir de forma complexa e interprofissional.

Foi um processo de construção coletiva no qual os envolvidos puderam oferecer e receber conhecimento, pois, ao passo que se construíam intervenções de forma coletiva, os participantes precisaram necessariamente desenvolver habilidades colaborativas, essenciais para o trabalho em saúde, tais como: escuta, comunicação, iniciativa, proatividade, sensibilidade, aprimoramento técnico, entre outras.

A partir disso, a participação no projeto possibilitou que os atores do PET assumissem novas formas de sentir, pensar e agir diante da comunidade com a qual se vincularam, passando a compreender o fazer saúde como um ato necessariamente coletivo. Tendo isso em mente, os resultados das intervenções futuras podem tornar-se cada vez mais eficientes, visto que compreender a complexidade das comunidades é o primeiro passo na construção de intervenções em saúde. É exigido o desenvolvimento de uma percepção ampliada como forma de acolher, acompanhar, conhecer e intervir de forma positiva na vida dos usuários.

Tantos os usuários quanto a equipe colheram os frutos dessa proposta de trabalho, pois foi uma oportunidade dos profissionais e dos acadêmicos estreitarem os vínculos entre as profissões ou cursos e enriquecerem os seus conhecimentos acerca do usuário que foi atendido, podendo, assim, ofertar uma assistência mais ampliada, integral e equânime.

\section{Referências}

Cervato-Mancuso, A. M., Vincha, K. R. R., \& Santiago, D. A. (2016). Educação alimentar e nutricional como prática de intervenção: reflexão e possibilidades de fortalecimento. Physis. 25 (1).

Coelho. F., \& Savassi, L. (2012). Aplicação de escala de risco familiar como instrumento de organização das visitas domiciliares. RBMFC, 1 (2), $19-26$.

Costa, M. V. (2016). The interprofessional education in Brazilian context: some reflections. Interface Comun Saúde Educ, 20 (56),197-8.

Costa, M. V., Peduzzi, M., Freire Filho, J. R. \& Silva, C. B. G. (2018). Educação interprofissional em saúde. SEDIS-UFRN.

Ferreira, L. A. (2019). Uso das práticas integrativas e complementares na estratégia saúde da família: cenário de um município de médio porte. 70f. Dissertação (Mestrado Profissional em Saúde da Família) - Centro de Ciências da Saúde, Universidade Federal do Rio Grande do Norte, Natal.

Guraya, S. Y., \& Barr, H. (2018). The effectiveness of interprofessional education in healthcare: A systematic review and meta-analysis. Kaohsiung Journal of Medical Sciences, 34(3), 160-165.

Lima, A. W. S. et al (2020). Perception and manifestation of collaborative competencies among undergraduate health students. Revista Latino-Americana de Enfermagem, 28.

Machado, S. K. K. et al. (2019) Relato de experiência PET-Saúde/interprofissionalidade. Seminário Integrador de Extensão, 2 (2).

Montenegro, A. V. (2013) Pet-saúde: relato de experiência estudantil na atenção básica. Extensão em ação, 3 (1).

Novack, V. C., Regente, T. L., Bim, C. R., \& Carrasco, A. C. (2020). Auriculoterapia: efeitos na ansiedade, sono e qualidade de vida. Revista Inspirar Movimento \& Saude, 20 (3), 1-15.

Organização Mundial da Saúde (2010). Marco para ação em educação interprofissional e prática colaborativa. Genebra: OMS.

Riskiyana, R., Claramita, M., Rahayu, G. R., \& Riskiyana, R. (2018). Objectively measured interprofessional education outcome and factors that enhance program effectiveness: A systematic review. Nurse Educ Today, 66,73-8.

Silva, V. G., Ribeiro, T. C., Machado, A. D., Duarte, S. J. H., Faria, C. et al. (2012). A educação física no programa de educação pelo trabalho para a saúde de Cuiabá-MT. Revista Bras Ativ Fis e Saúde, 17 (2), 121-124.

Soares, L. C., Santana, M. G., Thofehrn, M. B., \& Dias, D. G. (2009). Educação em saúde na modalidade grupal: relato de experiência. Ciência, Cuidado e Saúde, 8 (1), 118-123.

Vilaça, S. P. de O., \& Coutinho, D. J. G. (2019). Auriculoterapia no tratamento da obesidade na atenção básica. Revista Ibero-Americana de Humanidades, Ciências e Educação, 5 (3), 17.

Wiese, L., Gruber Ostrovski, E., Stefhani Keil, E., Ravache Keunecke, F., Barboza, J., \& Raquel Reichemback Danski, V. (2020). Projeto de Extensão riscos da automedicação: relato de experiências em educação em saúde. Extensão Tecnológica: Revista De Extensão Do Instituto Federal Catarinense, 7 (13), 64-88. 